\title{
Micro-optical Realization of Arrays of Selectively Addressable Dipole Traps: A Scalable Configuration for Quantum Computation with Atomic Qubits
}

\author{
R. Dumke, M. Volk, T. Müther, F. B. J. Buchkremer, G. Birkl, and W. Ertmer \\ Institut für Quantenoptik, Universität Hannover, Welfengarten 1, D-30167 Hannover, Germany
}

(Received 24 October 2001; published 8 August 2002)

\begin{abstract}
We experimentally demonstrate novel structures for the realization of registers of atomic qubits: We trap neutral atoms in one- and two-dimensional arrays of far-detuned dipole traps obtained by focusing a red-detuned laser beam with a microfabricated array of microlenses. We are able to selectively address individual trap sites due to their large lateral separation of $125 \mu \mathrm{m}$. We initialize and read out different internal states for the individual sites. We also create two interleaved sets of trap arrays with adjustable separation, as required for many proposed implementations of quantum gate operations.
\end{abstract}

DOI: 10.1103/PhysRevLett.89.097903

PACS numbers: 03.67.Lx, 32.80.Pj, 42.50.-p

The dramatic progress in micro- and nanofabrication of the hardware for information technology will lead to the encoding of logical information on a single particle basis in the near future. Therefore, the quantum behavior of the physical carriers of information has to be considered. Moreover, quantum effects may provide qualitatively new modes for information processing, driving research in the field of quantum information processing [1].

Among the broad range of currently investigated approaches, important progress has been obtained with atom physical schemes. Entanglement and quantum gate operations have been achieved with trapped ions [2] or photons in cavity QED experiments [3]. Several schemes for quantum gates based on the direct interaction of neutral atoms have been proposed theoretically [4-7]. Still, there is an intensive search for appropriate systems allowing the experimental realization of these schemes.

Several requirements have to be fulfilled for the successful implementation of quantum computation [8], such as the scalability of the physical system, the capability to initialize and read out qubits, long decoherence times, and the existence of a universal set of quantum gates. These requirements are potentially met by setups which are based on miniaturized structures for trapping, guiding, and manipulating neutral atoms as they are investigated in the newly developing field of integrated atom optics or ATOMICS [9]. This approach draws its strength from the combination of the well developed techniques for the manipulation of atomic quantum states with the state-of-the-art manufacturing basis of micro- and nanofabrication. A number of groups use microfabricated charged and current carrying structures for this purpose [10-17].

As an alternative approach, we have proposed the application of microfabricated optical elements (micro-optical elements) and have developed a number of configurations for atom optics, atom interferometry, and quantum information processing $[18,19]$. We consider this approach to be extremely powerful since most of the techniques for manipulating atomic qubits are based on the interaction with light and since optical trapping potentials can be made state selective in a simple fashion as required for many quantum gate proposals [4-6].

In this paper we present the first experimental implementation of microfabricated optical systems for quantum computing purposes with atoms: Fig. 1 shows rubidium atoms trapped in one- and two-dimensional arrays of dipole traps created by a micro-optical system. More than 80 traps hold atoms in Fig. 1(a). Each trap can act as a memory site for quantum information encoded in the two hyperfine ground states of the atoms. Thus, the arrays can serve as registers of atomic qubits.

We obtain the trap arrays by employing a twodimensional array of spherical, diffractive microlenses
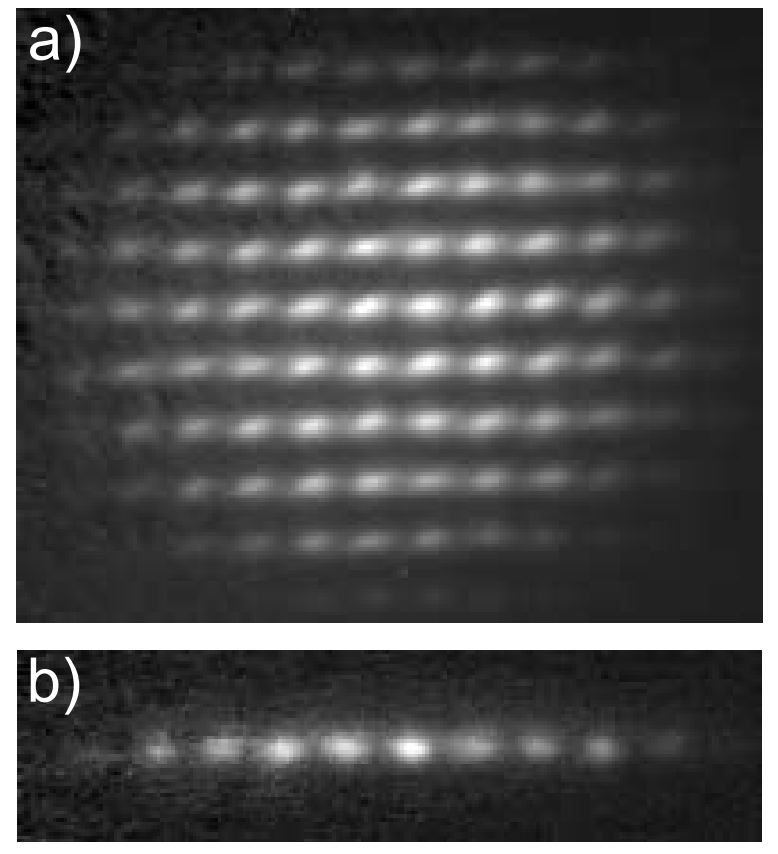

FIG. 1. (a) Two-dimensional and (b) one-dimensional arrays of rubidium atoms trapped in arrays of dipole traps. The traps are created using a micro-optical lens array and are separated by $125 \mu \mathrm{m}$. The brightest traps contain about $10^{3}$ atoms. 
with a focal length of $625 \mu \mathrm{m}$ and a lens diameter and separation of $125 \mu \mathrm{m}$ (Fig. 2). The microlens array is made of fused silica and contains $50 \times 50$ diffractive lenslets. The trapping light is derived from a $500 \mathrm{~mW}$ amplified diode laser system and is sent through a rubidium gas cell heated to a temperature of $110^{\circ} \mathrm{C}$ serving as a narrow band absorption filter. This reduces the strong background of amplified spontaneous emission by at least 2 orders of magnitude, otherwise preventing the operation of a dipole trap due to scattering of resonant photons. The light is then sent through an acousto-optical switching device and through a polarizer, which ensures a high degree of linear polarization. The remaining light [typical power $P=$ 100-200 mW, typical detuning $\Delta \lambda=0.2$ to $2 \mathrm{~nm}$ below the $5 S_{1 / 2}(F=3) \rightarrow 5 P_{3 / 2}\left(F^{\prime}=4\right)$ transition at $780 \mathrm{~nm}$ ("red detuning")] is focused by the microlens array. In order to have full optical access for atom preparation and detection, we image the focal plane of the microlens array onto a magneto-optical trap (MOT) with the help of two achromats (magnification $=1$ and no significant reduction of numerical aperture). Thus, we obtain an array of foci with a separation of $125 \mu \mathrm{m}$ and a spot size of $(7 \pm 2) \mu \mathrm{m}$ $\left(1 / e^{2}\right.$ radius of intensity). For a red-detuned laser beam, this results in an array of dipole traps, each analogous to a trap obtained by a single focused laser beam [20,21] (see also [22]). The optical transfer of the trapping light has the additional advantage that we can place the micro-optical system outside the vacuum chamber and thus can switch between a variety of micro-optical elements easily. On the other hand, trapping of atoms directly in the first focal plane close to the surface is possible as well.

We load the array of dipole traps and detect the trapped atoms similar to [23]: We start with a MOT of $10^{7}$ to $10^{8}$ ${ }^{85} \mathrm{Rb}$ atoms which we overlap for several hundreds of $\mathrm{ms}$ with the dipole trap array and optimize the loading process for the highest atom number. The MOT is then switched off, and the atoms are held in the dipole traps for a variable storage time (typically 25 to $60 \mathrm{~ms}$ ). This time is long enough for untrapped atoms to leave the detection region. The primary MOT light and the repumper are switched on

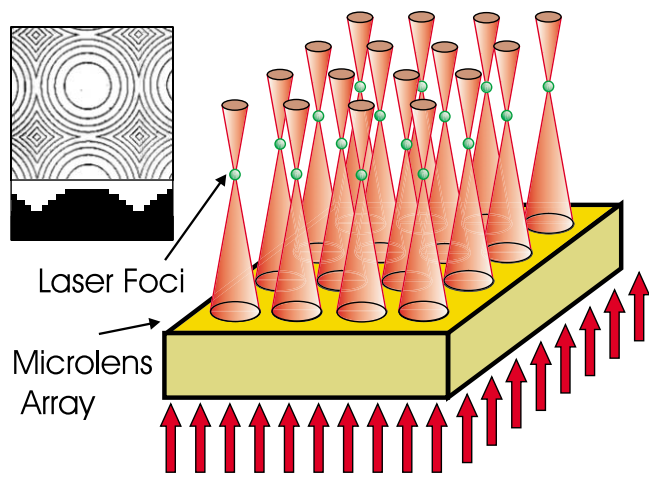

FIG. 2 (color online). A two-dimensional array of laser foci is created by focusing a single laser beam with an array of microlenses. Inset: Phase contrast image and typical cross section of a small part of a diffractive microlens array. again for a period of approximately $1 \mathrm{~ms}$ to detect the trapped atoms via spatially resolved detection of fluorescence with a spatial resolution of $17 \mu \mathrm{m}$ (rms spread of the smallest observed structures).

We obtain a two-dimensional array of approximately 80 well separated dipole traps with a potential depth of about $1 \mathrm{mK}$ containing up to $10^{3}$ atoms [Fig. 1(a)]. The number of filled traps is limited by the size of the laser beam illuminating the microlens array and by the initial MOT size. The apparent larger extent of the individual traps in the horizontal direction in all images presented in this paper is caused by the detection optics being horizontally tilted relative to the beam axis of the trap light, necessary to avoid trapping light entering the camera aperture. The detection efficiency of our setup is already high enough to be able to detect atom samples of fewer than 100 atoms per trap. We are currently optimizing the detection efficiency to allow the observation of single atoms as well $[21,24,25]$.

Illumination of only one row of the microlens array leads to a one-dimensional array of dipole traps [Fig. 1(b)]. For the traps of this array (power per trap $P=$ $3 \mathrm{~mW}, \Delta \lambda=0.4 \mathrm{~nm}$ ) the calculated potential depth is $U_{0} / k_{B}=2.5 \mathrm{mK}$, which agrees within a factor of 2 with the one inferred from the measured radial oscillation frequency of $7.5 \mathrm{kHz}$ [26]. The discrepancy can be fully explained by the known uncertainties in determining the laser power per trap, the focal waist, and the oscillation frequencies. The lifetime of the atoms in the traps is $35 \mathrm{~ms}$, which is most probably limited by heating due to scattering of residual near-resonant light not completely absorbed from the trapping beam. Using a time-of-flight technique, we determined the atom temperature to be below $20 \mu \mathrm{K}$, which suggests the presence of an additional cooling mechanism during the loading phase as is also observed in [27].

In addition to its scalability, our approach is especially suited to fulfill another requirement for the physical implementation of quantum information processing, namely, the ability to selectively address, initialize, and read out individual qubits: The large lateral separation between the dipole traps enables us to selectively address the individual traps in a straightforward fashion. We demonstrate this by focusing a near-resonant laser beam onto one of the dipole traps for a few ms after the loading process is completed [28]. This heats the atoms out of the addressed dipole trap. As can be seen in Fig. 3, no atoms are left at the site of the addressed dipole trap, while the atoms at the adjacent sites remain unaffected. By two-dimensional scanning of the addressing beam or by illuminating each lenslet individually with spatially modulated addressing light, every site can be addressed individually. This opens the possibility to selectively prepare and manipulate the qubits in the individual traps.

As a next step, we demonstrated the site specific and state selective initialization and readout of atomic quantum states (Fig. 4). Here we illuminate a one-dimensional atom 


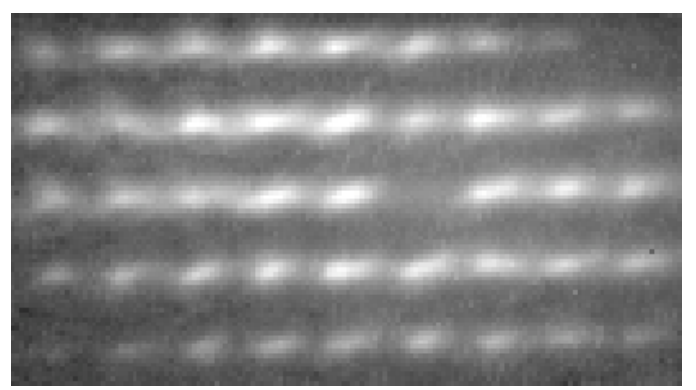

FIG. 3. Demonstration of the selective addressability of individual trap sites: By focusing a near-resonant laser beam onto one of the dipole traps (row 3, column 6) during the storage period, the atoms in this trap are removed, while the other dipole traps remain unaffected.

array [analogous to Fig. 1(b)] only with light resonant with the $5 S_{1 / 2}(F=3) \rightarrow 5 P_{3 / 2}\left(F^{\prime}=4\right)$ transition (i.e., the repump light switched off) during detection. Since the atoms are almost exclusively in the lower hyperfine ground state $5 S_{1 / 2}(F=2)$ after the loading phase, they do not scatter the detection light [Fig. 4(a)] unless we actively pump them into the upper hyperfine ground state $5 S_{1 / 2}(F=3)$ during the time the atoms are stored in the dipole traps, i.e., prior to the detection phase [Figs. 4(b) and 4(c)]. This has been done for one [Fig. 4(b)] or, alternatively, all [Fig. 4(c)] of the trap sites. This demonstrates the site specific and state selective initialization and detection capability of our approach.

While many of the advantages of our system result from the large lateral separation of the individual trap sites, it is also possible to actively control the distance between individual traps if smaller or adjustable distances are required, e.g., for quantum gate operations and the entanglement of atoms via atom-atom interactions. In our setup, this can be accomplished by illuminating one microlens array with two beams under slightly different angles
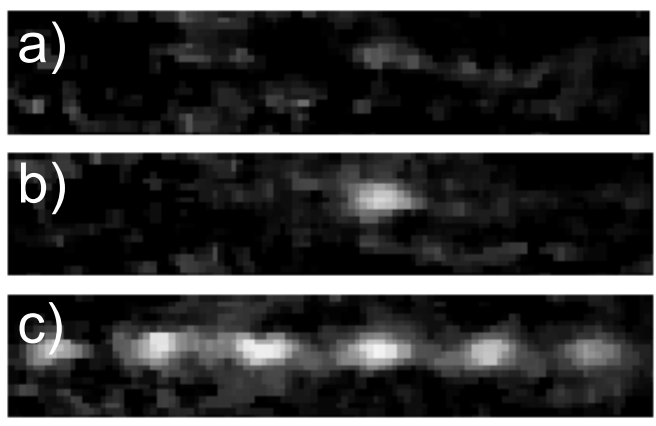

FIG. 4. Site specific and state selective initialization and readout of quantum states. All sites of a one-dimensional array of dipole traps are filled with atoms. For detection only light resonant with the $5 S_{1 / 2}(F=3) \rightarrow 5 P_{3 / 2}\left(F^{\prime}=4\right)$ transition is applied. (a) All atoms are in the $F=2$ state; they do not scatter the detection light. (b) The atoms at one site have been transferred to the $F=3$ state and can be seen. (c) The atoms at all sites have been transferred to the $F=3$ state.
[Fig. 5 (left)] [18,21], which results in two interleaved sets of arrays of trapped atoms. Interference effects between the two laser beams are prevented by using orthogonal linear polarizations. Figure 5 (right) shows two vertically displaced sets of arrays of trapped atoms with a mutual separation of $45 \mu \mathrm{m}$. The separation depends only on the angle between the two laser beams and can easily be changed, especially to smaller values. By reducing the relative angle to zero, overlapping traps can be created. Figure 6 shows vertical cross sections through one pair of dipole traps for different angles between the two laser beams and thus different site separations. We could also change the separation of the traps and thus move the atoms in one set of traps in real time by deflecting one beam with the help of a fast acousto-optical beam deflector. With this technique it should become possible to selectively prepare qubits at large distances, then decrease the distance for gate operations and entanglement via atom-atom interactions, and then increase the distance again for the readout of the qubit states.

In order to demonstrate the full potential for quantum information processing, we evaluate the criteria given in [8] for our approach. Scalability, site specific and state selective initialization, and readout, as well as the ability to change the separation of trapping sites at will, are the most important advantages of our approach, and have been demonstrated in the previous sections. In addition, the concept presented here and the underlying technology fulfill the remaining two criteria, namely, long relevant decoherence times and the availability of a universal set of quantum gates as well.

With the specific lens array of this work (beam waist $7 \mu \mathrm{m}$ ) and a standard set of laser parameters (Ti:Sa laser, wavelength $825 \mathrm{~nm}$, power per trap $50 \mathrm{~mW}$ ) several tens of traps with radial vibrational frequencies of $10 \mathrm{kHz}$ and a decoherence time (assumed to be limited by spontaneous scattering) of $50 \mathrm{~ms}$ can be obtained [18]. These parameters allow the implementation of collisional gates [4] with a decoherence time being at least 50 times longer than the gate time.

The full potential of our concept can be exploited by using available lens arrays optimized for large numerical aperture: it has been shown experimentally [29] that beam
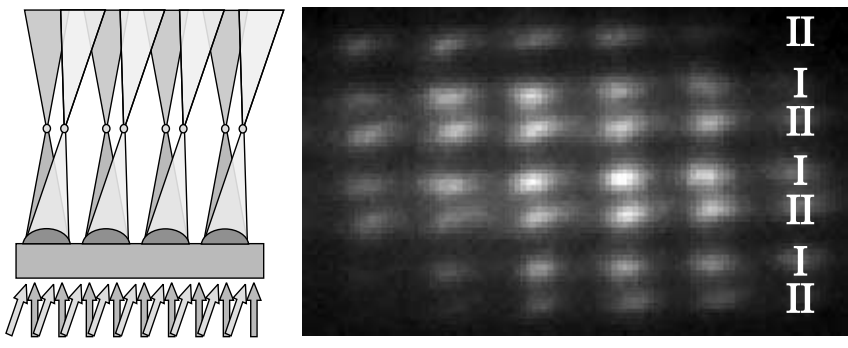

FIG. 5. Two interleaved sets of arrays of trapped atoms (arrays I and II at right) obtained by illuminating one microlens array with two beams under slightly different angles (left). The distance between traps of the two arrays is approximately $45 \mu \mathrm{m}$. 


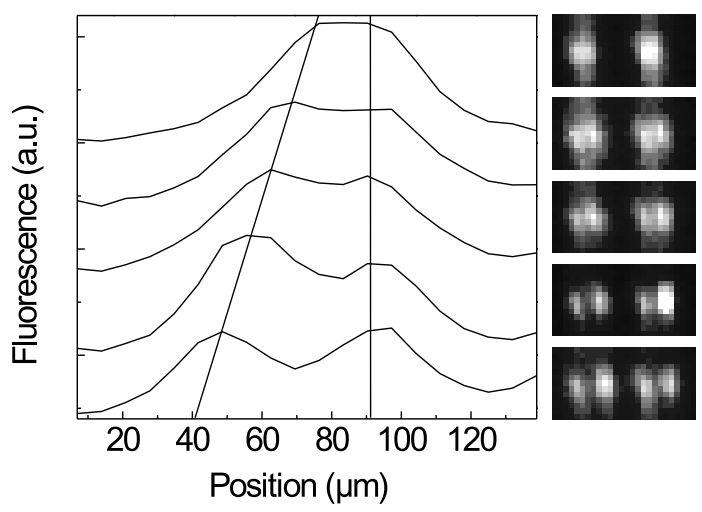

FIG. 6. (Left) Cross sections through pairs of dipole traps as in Fig. 5 (rotated by $90^{\circ}$ ) for different separations between the traps ranging from 18 to $45 \mu \mathrm{m}$. (Right) Image sections showing two pairs of traps with decreasing separation from bottom to top corresponding to the cross sections at left.

waists below $1 \mu \mathrm{m}$ can be achieved with microlens arrays similar to the one used in this work. With standard laser parameters (wavelength $850 \mathrm{~nm}$, power per trap $1 \mathrm{~mW}$ ) radial vibrational frequencies of $50 \mathrm{kHz}$ and decoherence times of $150 \mathrm{~ms}$ can be obtained. This allows the implementation of various types of quantum gates $[4,6,7]$. Because of its potentially short gate times and its insensitivity to the temperature of the atoms and to the variations in atom-atom separation, the Rydberg gate of [6] deserves specific attention: Using the parameters and scaling laws of [6], for atoms in microlens dipole traps with a waist and a respective minimum trap separation of $1 \mu \mathrm{m}$, a gate time of about $1 \mu \mathrm{s}$ can be achieved. This gate time is 20 times shorter than the oscillation period and $10^{4}$ times shorter than the decoherence time, giving favorable experimental conditions. Further evidence for the potential of this approach can be drawn from the fact that this array of $1 \mu \mathrm{m}$-waist dipole traps represents a $2 \mathrm{D}$ extension of the dipole trap demonstrated in [21]. For this trap a detailed investigation [30] gives proof for the feasibility of Rydberg gates and explicitly shows that for trap separations of 1 to $5 \mu \mathrm{m}$, gate times of 1 to $10 \mu \mathrm{s}$ are possible.

We conclude this discussion by pointing out that since our dipole trap arrays can give the same trap parameters as single dipole traps or standing wave dipole potentials, the single atom loading schemes experimentally demonstrated in [21] and [25] can be extended to our configurations in a straightforward fashion. In addition, with the achievable vibrational frequencies being larger than the recoil frequency, sideband cooling of atoms to the vibrational ground state of the trap arrays should be possible. Finally, the demonstration of the loading of periodic dipole potentials with single atoms from a Bose-Einstein condensate [31] might allow an extemely efficient means of single atom loading into vibrational ground states of our trap arrays.

This work is supported by the SFB 407 and the program Quanteninformationsverarbeitung of the Deutsche Forschungsgemeinschaft, as well as by the program
ACQUIRE (IST-1999-11055) of the European

Commission.

[1] A. M. Steane, Rep. Prog. Phys. 61, 117 (1998); The Physics of Quantum Information, edited by D. Bouwmeester, A. Ekert, and A. Zeilinger (Springer, Berlin, 2000); Special Issue of Fortschr. Phys. 48 (2000).

[2] C. Monroe et al., Phys. Rev. Lett. 74, 4011 (1995); C. A. Sackett et al., Nature (London) 404, 256 (2000).

[3] E. Hagley et al., Phys. Rev. Lett. 79, 1 (1997); A. Rauschenbeutel et al., Phys. Rev. Lett. 83, 5166 (1999).

[4] D. Jaksch et al., Phys. Rev. Lett. 82, 1975 (1999); T. Calarco et al., Phys. Rev. A 61, 022304 (2000).

[5] G. K. Brennen et al., Phys. Rev. Lett. 82, 1060 (1999).

[6] D. Jaksch et al., Phys. Rev. Lett. 85, 2208 (2000).

[7] E. Charron, E. Tiesinga, F. Mies, and C. Williams, Phys. Rev. Lett. 88, 077901 (2002).

[8] D. P. DiVincenzo, Fortschr. Phys. 48, 9 (2000).

[9] For atom optics with microstructures.

[10] J. D. Weinstein and K. G. Libbrecht, Phys. Rev. A 52, 4004 (1995); J. Schmiedmayer, Eur. Phys. J. D 4, 57 (1998); E. A. Hinds and I. G. Hughes, J. Phys. D 32, R119 (1999).

[11] J. Reichel, W. Hänsel, and T. W. Hänsch, Phys. Rev. Lett. 83, 3398 (1999); W. Hänsel, J. Reichel, P. Hommelhoff, and T.W. Hänsch, Phys. Rev. Lett. 86, 608 (2001); W. Hänsel, P. Hommelhoff, T.W. Hänsch, and J. Reichel, Nature (London) 413, 498 (2001).

[12] D. Müller et al., Phys. Rev. Lett. 83, 5194 (1999).

[13] N. H. Dekker et al., Phys. Rev. Lett. 84, 1124 (2000).

[14] M. Key et al., Phys. Rev. Lett. 84, 1381 (2000).

[15] R. Folman et al., Phys. Rev. Lett. 84, 4749 (2000); D. Cassettari et al., Phys. Rev. Lett. 85, 5483 (2000).

[16] H. Ott et al., Phys. Rev. Lett. 87, 230401 (2001).

[17] P. Engels, W. Ertmer, and K. Sengstock, Opt. Commun. 204, 185 (2002).

[18] G. Birkl, F. B. J. Buchkremer, R. Dumke, and W. Ertmer, Opt. Commun. 191, 67 (2001).

[19] F. B. J. Buchkremer et al., Laser Phys. 12, 736 (2002).

[20] For an overview see R. Grimm, M. Weidemüller, and Y. B. Ovchinnikov, Adv. At. Mol. Opt. Phys. 42, 95 (2000).

[21] N. Schlosser, G. Reymond, I. Protsenko, and P. Grangier, Nature (London) 411, 1024 (2001).

[22] D. Boiron et al., Phys. Rev. A 57, R4106 (1998).

[23] S. J. M. Kuppens et al., Phys. Rev. A 62, 013406 (2000).

[24] Z. Hu and H. J. Kimble, Opt. Lett. 19, 1888 (1994); F. Ruschewitz et al., Europhys. Lett. 34, 651 (1996); D. Frese et al., Phys. Rev. Lett. 85, 3777 (2000).

[25] S. Kuhr et al., Science 293, 278 (2001).

[26] We measure this frequency by parametrically heating the atoms and observing the number of remaining atoms.

[27] M. D. Barrett, J. A. Sauer, and M. S. Chapman, Phys. Rev. Lett. 87, 010404 (2001).

[28] R. Scheunemann, F. S. Cataliotti, T. W. Hänsch, and M. Weitz, Phys. Rev. A 62, 051801 (2000).

[29] T. Hessler et al., Pure Appl. Opt. 6, 673 (1997).

[30] I.E. Protsenko, G. Reymond, N. Schlosser, and P. Grangier, Phys. Rev. A 65, 052301 (2002).

[31] M. Greiner, O. Mandel, T. Esslinger, T. W. Hänsch, and I. Bloch, Nature (London) 415, 39 (2002). 